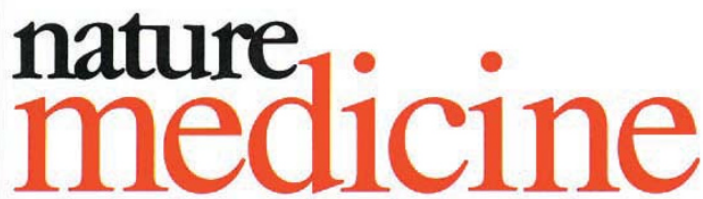

VOLUME 3 • NUMBER 12 • DECEMBER 1997

\title{
Editors and ethics
}

Biomedical research journals are seeing a flood of competitive and cutting edge research that challenges accepted protocol and ethical practices. Whether it is funding sources that may carry a conflict of interest, patents with ill defined applications or procedures such as xenotransplantation, cloning or human fetal research, journal editors need a policy on how to handle ethical questions. Many researchers see editors as gatekeepers or, even worse, as members of a quasi ethics police force. But editors should not be tempted either to set ethical standards or to take on the role of enforcers.

The peer review process unearths details of the background from which each published paper grew: Who instigated the work; Why and what was the rationale for the approach taken; Who funded it and what was their motive; Who is included as an author? These and other issues may give rise to ethical concerns and one gets the impression that the incidences of such concerns is on the rise. Although there are no statistics to confirm this perception, it does seem reasonable to suggest that biomedical research is becoming more competitive and aggressive. With much more at stake, the "publish or perish" mentality is dominant and, so it seems, quite overwhelming for some who start to bend the rules and may even resort to out-and-out fraud. Certainly, governments, research agencies and universities think that the problem is sufficient to warrant the establishment of education programs and investigative bodies.

From the editors perspective, scientific fraud-a deliberate deception-is the easiest issue to deal with. If the fraud is convincing and well executed, editors and expert referees will not notice it and therefore there is nothing really to be done. An often forgotten but logical extension of this is that the editors of journals do not verify any of the work that they publish. The rationale behind the research, its presentation and the interpretation of the work are examined, but the actual raw data is rarely seen and even if it were, this would do nothing to verify the integrity and origin of the research. Science is based on trust and as with all such endeavors, it is not difficult to cheat if that is your intention.

In other instances, less dramatic than out-and-out fraud, scientific misconduct may give rise to disputes that editors are asked to resolve such as authorship disputes, or the selective presentation of data. Should editors confront authors with such concerns or ask them to verify certain claims? The simple answer is no. Although it could be argued that journals do have the authority to confirm basic claims in a paper, they certainly have no mandate. Nor from a practical point of view can they confirm ethical behavior or sanction what might be less than ethical.

Perhaps the more demanding ethical issues are those involving new paradigms and applications of technology, as, by definition, there are no precedents to look to. How should journals react to work that actually satisfies that over-used cliché, and does "push back frontiers"?

Of course, if someone submits a research paper in which it is quite clear that a norm of research ethics has been trampled, a journal could, and should, refrain from publishing it, citing specific concerns. However, that is rare and it is much more likely that when a paper takes a bold step in a new direction, it will not appear to challenge existing ethical guidelines or simply fails to mention whether accepted ethical standards and codes were honored. Are journals expected to question the authors of all such articles in an attempt to confirm ethical practices? If the answer is yes then we must prepare ourselves for a lot of questioning because the simple truth is that most authors do not address such issues at the time of submission. And more often than not, those that do, simply regurgitate a standard sentence that to all purposes is a formality and on which little significance can be placed. A further level of complexity is added if we take into account the geographical (and therefore cultural) origin of the paper. Some institutions will not fund human embryo research, whereas others will-it is impossible for editors to know all these occasions and act accordingly.

The point is two-fold: First, the time for addressing ethical concerns with biomedical research is not when the work is completed and submitted for publication, but before it is begun. Secondly, journal editors should not be gatekeepers or judges, deciding what does and does not fulfill ethical criteria. This is not a case of ducking responsibility. Editors do have to be informed and active in the arena of bioethics and they should be prepared to contribute to the debate and to support commonly agreed standards. They should also encourage debate and consensus ahead of time-germ line gene therapy, for example, is just around the corner and the community should not be waiting for submission of the first manuscript, before formulating guidelines on the moral and ethical arguments for and against such work. But editors should contribute no more or less than the other members of society. If the work is not ethical, it should have been stopped long before submission for publication. We must find a longer-term approach to educating scientists and medics of their ethical obligations, such that publication is never the issue. 